\title{
Low Trap Density in InAs/High-k Nanowire Gate Stacks with Optimized Growth and Doping Conditions
}

\author{
Jun Wu, Aein Shiri Babadi, Daniel Jacobsson, Jovana Colvin, Sofie Yngman, Rainer Timm, \\ Erik Lind, and Lars-Erik Wernersson
}

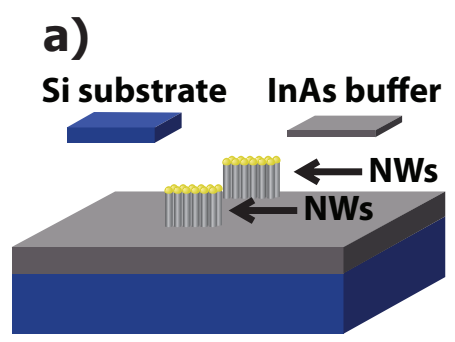

d)

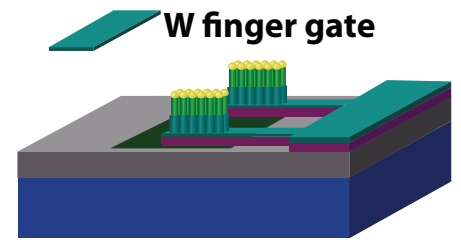

b)

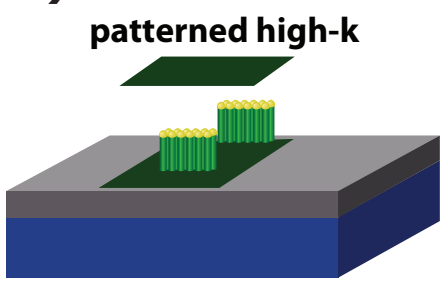

e)

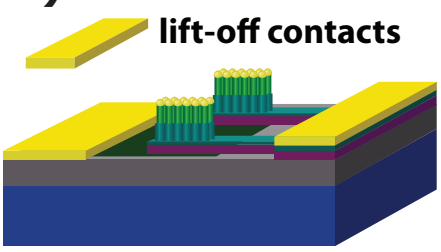

c)
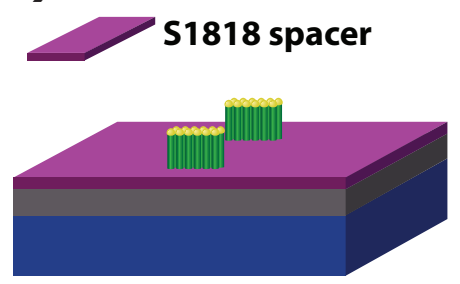

f) buffer layer mesa etching

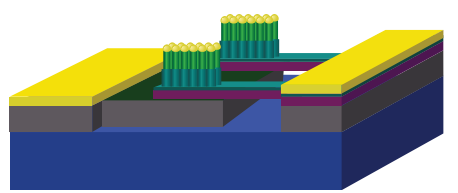

Figure S1. Schematic layout of the vertical wrap-gated nanowire MOS capacitor processing: a) Nanowire growth on the (111) Si substrate with a thin InAs buffer layer; b) ALD high- $k$ deposition, followed by UV lithography patterning; c) S1818 spacer layer deposition, followed by RIE etching to define the thickness of about $150 \mathrm{~nm}$; d) W finger contact patterning using deep UV lithography and EBL; e) Ti/W/Au pad deposition by lift-off; f) Buffer layer mesa etching. 




b)
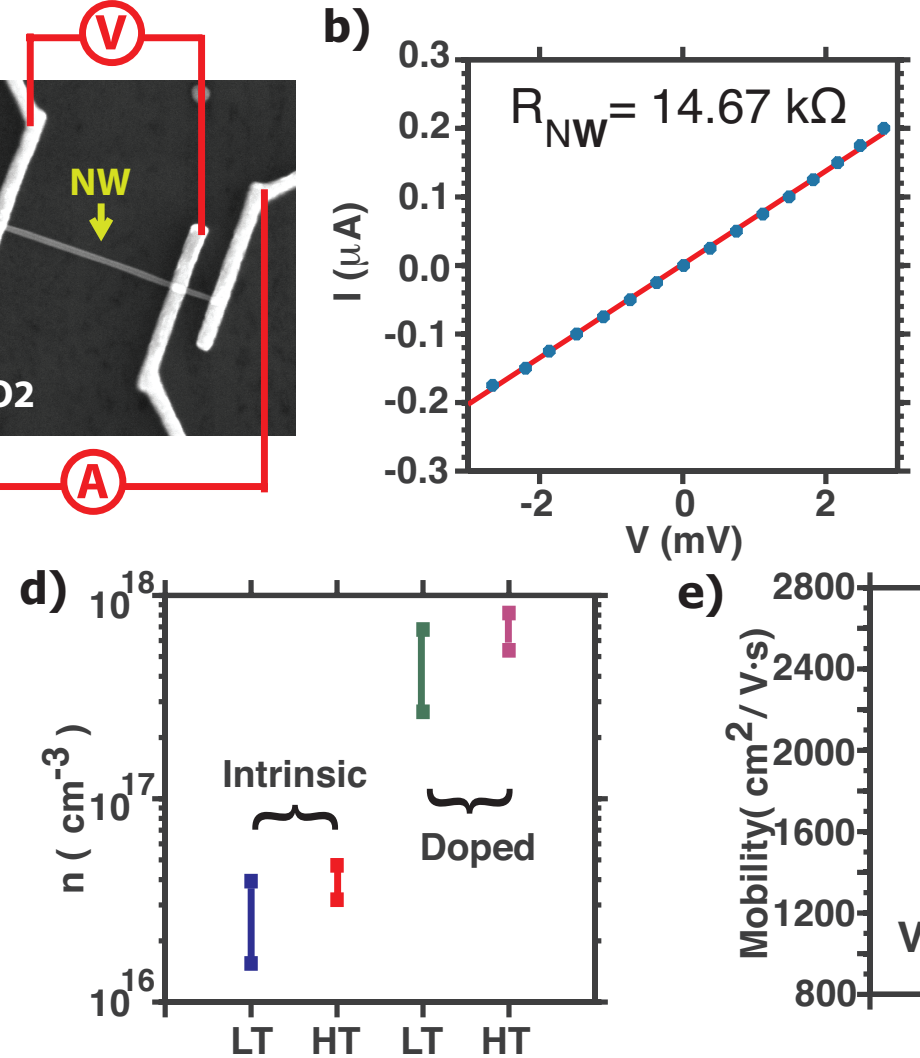

e) c)

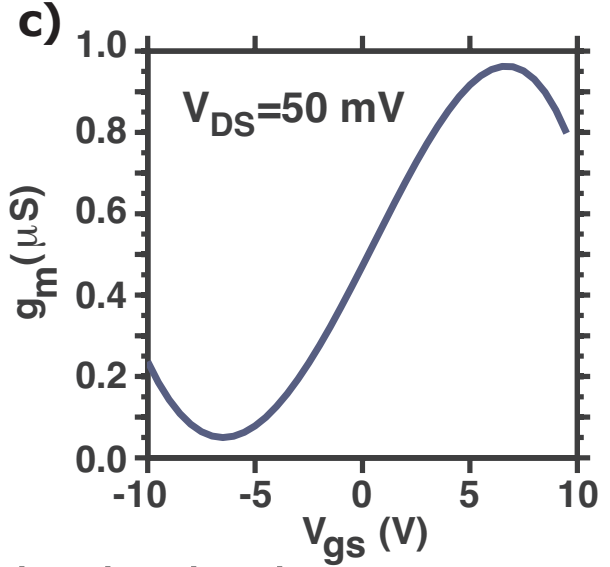



Figure S2. a) Fabricated four-probe back-gate lateral nanowire field-effect transistor used for transport measurements. The figure also illustrates how the four-probe current-voltage (I-V) measurement is performed. The current is applied to the two outer electrodes, while the voltage drop between the two inner electrodes is read. b) Measured (blue dots) and fitted (red line) I-V curves for an intrinsic nanowire. c) Measured transconductance $g_{m}=\partial I / \partial V_{G S}$ at drain-source voltage of $50 \mathrm{mV}$. d)-e) show the extracted effective mobile carrier concentrations, $\mathrm{n}$, and mobilities, $\mu_{n}$, for nanowires in Set I (intrinsic) and Set II (doped), respectively. 


\section{b)}

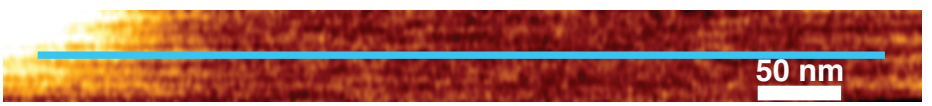

a)


Figure S3. AFM images of nanowires from the LT shell sample, laterally deposited onto an InAs substrate. a) 3D rendered $800 \mathrm{~nm} \times 800 \mathrm{~nm}$ overview image showing several nanowires. Height scale is $80 \mathrm{~nm}$. Note that the appearance of the nanowires is a convolution of the actual nanowire profile and the AFM tip shape. b), d) AFM images on top of a nanowire side facet, the AFM scan direction (long dimension) is parallel to the nanowire growth direction. c), e) Height profiles along the nanowire growth direction, obtained from the AFM images, as indicated by the blue lines in b), d). The nanowire imaged in b), c) shows some tapering at one end and an otherwise flat surface, while the nanowire imaged in d), e) features two surface steps with a height of two atomic monolayers. 

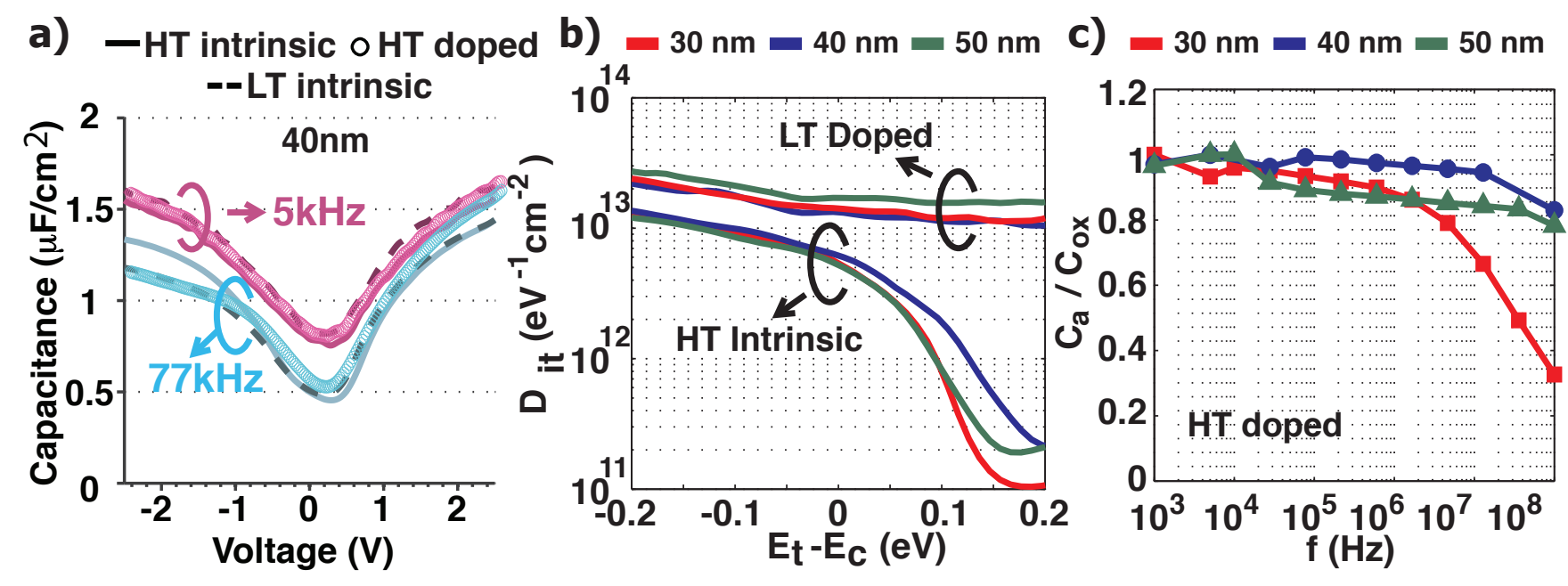

Figure S4. a) Comparison of $\mathrm{C}-\mathrm{V}$ characteristics between the intrinsic nanowire MOS capacitors and the HT doped nanowire MOS capacitor. It shows similar $\mathrm{C}-\mathrm{V}$ modulations between the devices, indicating the gate stack quality is not severely degraded by the doping for HT grown nanowire MOS capacitors. b) Comparison of $D_{i t}$ profiles for nanowires with different diameters. Here only the results for the HT intrinsic and LT doped devices are shown. The same trend is also observed for all the other cases within the diameter range studied in the work. c) Normalized accumulation capacitances as a function of the measurement frequency for HT doped nanowire MOS capacitors with different nanowire diameters. The figure indicates a diameter dependent doping level for these devices with smaller diameter nanowire incorporating less dopant, hence resulting in larger series resistance and lower transition frequency. No such dependence is observed for LT doped devices within the measurement frequency range. 